\title{
A Influência de Parâmetros Atmosféricos na Contagem de Radiação X e Gama de Fundo
}

Mauro A. Alves ${ }^{1}$, Inácio M. Martin ${ }^{1}$, Alessandro J. de Abreu ${ }^{1}$ e Anatoli Gusev ${ }^{2}$

${ }_{1}^{1}$ Instituto Tecnológico de Aeronáutica, Divisão de Ciências Fundamentais, São José dos Campos, SP, Brasil

${ }^{2}$ Space Research Institute of the Russian Academy of Sciences, Moscow, Russia

Copyright 2016, SBGf - Sociedade Brasileira de Geofísica

Este texto foi preparado para a apresentação no VII Simpósio Brasileiro de Geofísica, Ouro Preto, 25 a 27 de outubro de 2016. Seu conteúdo foi revisado pelo Comitê Técnico do VII SimBGf, mas não necessariamente representa a opinião da SBGf ou de seus associados. É proibida a reprodução total ou parcial deste material para propósitos comerciais sem prévia autorização da SBGf.

\section{Resumo}

Time series of background $X$ and gamma radiation counts, precipitation and wind speed and direction were collected to determine whether variations in these series are correlated. Data collected during a period of 162 days with a one-minute sampling ratio were analyzed using Fast Fourier Transform, correlation analysis and wavelet transform. The results show a clear enhancement of the background radiation associated with rainfall caused by radon washout, but do not show any measurable effect of the wind on the radiation counts.

\section{Introdução}

É um fato bem conhecido que o gás radônio, produzido através do decaimento do urânio e tório presentes no solo, rochas e crosta terrestre se difunde na atmosfera como resultado dos processos de exalação e emanação. São conhecidos 40 isótopos radioativos do radônio. Quatro radioisótopos do radônio $\left({ }^{222} \mathrm{Rn}, \mathrm{t}_{1 / 2}=3,8235 \mathrm{~d}\right.$; ${ }^{220} \mathrm{Rn}, \mathrm{t}_{1 / 2}=55,6 \mathrm{~s} ;{ }^{219} \mathrm{Rn}, \mathrm{t}_{1 / 2}=3,96 \mathrm{~s}, \mathrm{e}{ }^{218} \mathrm{Rn}, \mathrm{t}_{1 / 2}=35$ $\mathrm{ms}$ ) são formados naturalmente e encontrados em quantidades vestigiais no meio ambiente (McGill et al., 2015). Desses, o ${ }^{222} \mathrm{Rn}$ é o mais significante em termos de sua contribuição à radiação de fundo e aspectos relacionados à saúde humana em função de sua meiavida mais longa, o que permite que ele migre por distâncias relativamente grandes no ambiente geológico antes de decair. (Groves-Kirkby et al., 2006; Denman et al. 2004). Ao escapar do solo, o ${ }^{222} \mathrm{Rn}$ é misturado à atmosfera por processos turbulentos, mas devido à sua densidade, a sua concentração decresce com a altura (Willians et al., 2011). Durante a sua permanência na atmosfera, o ${ }^{222} \mathrm{Rn}$ decai em seus descendentes radioativos de acordo com a cadeia de decaimento:

${ }^{222} \mathrm{Rn} \rightarrow{ }^{218} \mathrm{Po} \rightarrow{ }^{214} \mathrm{~Pb} \rightarrow{ }^{214} \mathrm{Bi} \rightarrow{ }^{214} \mathrm{Po} \rightarrow{ }^{210} \mathrm{~Pb}$

onde $t_{1 / 2}\left({ }^{218} \mathrm{Po}\right)=3,1 \mathrm{~min}, \mathrm{t}_{1 / 2}\left({ }^{214} \mathrm{~Pb}\right)=26,8 \mathrm{~min}, \mathrm{t}_{1 / 2}\left({ }^{214} \mathrm{Bi}\right)$ $=20 \mathrm{~min}, \mathrm{t}_{1 / 2}\left({ }^{214} \mathrm{Bi}\right)=164 \mu \mathrm{s}$ (a meia vida do ${ }^{210} \mathrm{~Pb}$ é 22,2 anos, portanto muito longa para ser considerada no presente estudo; Wilkening, 1990). Na atmosfera, os descendentes do ${ }^{222} \mathrm{Rn}$ podem ser adsorvidos por aerossóis, partículas de poeira ou gotículas de água.
Através da precipitação, o ${ }^{222} \mathrm{Rn}$ e seus descendentes são removidos da atmosfera pelo processo conhecido como radon washout (Bandhari \& Rama, 1963). Esse processo, como indica o nome, consiste na absorção por gotas de chuvas desses elementos radioativos que são depositados no solo ou então transportados para as regiões mais baixas da atmosfera, no caso do ${ }^{222} \mathrm{Rn}$. Um efeito do radon washout que pode ser registrado com relativa facilidade é o aumento da radioatividade ambiente. No decaimento dos radioisótopos da cadeia (1) ocorre a emissão de radiação gama que remove o excesso de energia dos radioisótopos. Recentemente, foi demonstrado que curvas de variação de contagem de radiação gama durante eventos de radon washout podem ser simuladas levando em conta o decaimento radioativo dos radioisótopos na cadeia (1) (Gusev et al., 2015). Esse resultado indica que a observação e registro da radiação gama ambiental podem ser utilizados como um método indireto para determinar a presença de radônio no ambiente e variações de sua concentração. Sem dúvida, efeitos meteorológicos podem contribuir de maneira importante na variação da concentração desse gás, como mencionado anteriormente a chuva pode ocasionar o aumento local e temporário da concentração desse radioisótopo. O objetivo desse estudo é determinar ser outra variável meteorológica como o vento pode causar um efeito inverso ao do radon washout, ocasionado a sua dissipação do radônio, diminuindo a sua concentração localmente. Para isso foram analisadas séries de tempo de contagens de radiação gama, precipitação e intensidade e direção do vento para um período de mais de cinco meses, coletadas na cidade de São José dos Campos, SP.

\section{Materiais e Métodos}

Contagens de radiação gama e $X$ no intervalo de energia de $30 \mathrm{keV}$ a $10 \mathrm{Mev}$ foram coletadas por um detector constituído por um cintilador de iodeto de sódio dopado com tálio [ $\mathrm{Nal}(\mathrm{TI})]$ (dimensões do cristal: diâmetro, 7,5 $\mathrm{cm}$; altura, $7,5 \mathrm{~cm}$ ) acoplado a uma fotomultiplicadora (Gamma Radiation Detector, Modelo 44-20 Ludlum, EUA). O cristal e a fotomultiplicadora estão alojados em uma carcaça de alumínio para proteção mecânica. A fotomultiplicadora é alimentada por uma fonte alta tensão de $1400 \mathrm{~V}$, gerada a partir de uma tensão de entrada de 12 VDC. Os sinais de pulsos de luz UV produzido pela interação entre a radiação gama e o cristal são amplificados e digitalizados por um sistema compacto e de baixo custo (Modelo PMI-30, AWARE Electronics EUA). Um software específico detecta os sinais 
digitalizados e os grava em computador para posterior análise. O detector e a eletrônica associada estavam abrigados em container com ar condicionado de tal forma que as contagens de raios gama foram efetuadas a uma temperatura constante $\left(22^{\circ} \mathrm{C}\right)$. Dados de precipitação e direção e intensidade do vento foram coletados por uma estação meteorológica automática (Modelo GRWS10, Campbell, EUA). O anemógrafo estava instalado em uma torre a uma altura de $5 \mathrm{~m}$ do solo. O experimento foi conduzido em São José dos Campos, SP $\left(23,212573^{\circ} \mathrm{S}\right.$, 45,866568 " W; altitude, $620 \mathrm{~m}$ ), no interior do campus do Departamento de Ciência e Tecnologia Aeroespacial (DCTA) na área da Torre de Observação de Fenômenos Atmosféricos do Instituto Aeronáutico e Espaço, Divisão de Ciências Atmosféricas (TOFA-IAE/ACA). O local onde os instrumentos estavam instalados é um espaço aberto, sem presença nas imediações de afloramentos rochosos, e reservado para a obtenção de medidas meteorológicas e ambientais. Nesse local não existem construções ou estruturas capazes de interferir com a liberação ou causar a retenção do gás radônio, Fig.1. O tipo de solo na região é areno-argiloso formado por sedimentos terciários da bacia do Rio Paraíba do Sul (Vale do Paraíba), e o substrato rochoso na região é formado por gnaisses ortoderivados (CPRM 2010).

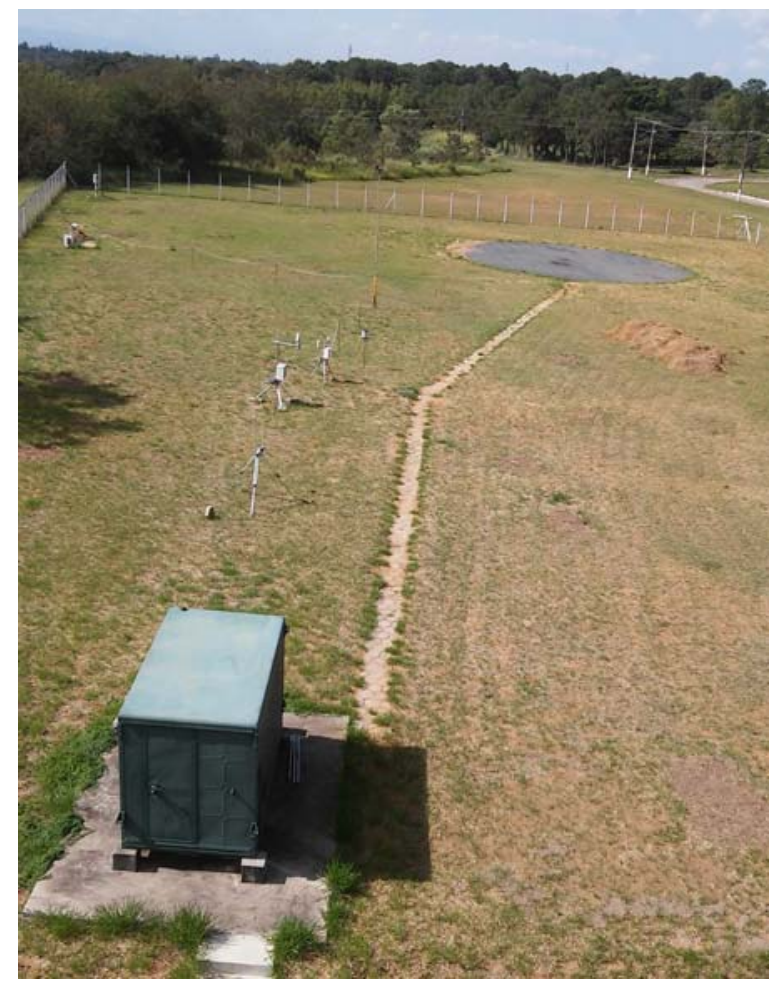

Figura 1 - Local onde foi realizado o estudo. O container abriga o cintilador e eletrônica associada; os instrumentos meteorológicos podem ser observados no plano de fundo.
A coleta das séries de tempo for realizada de maneira ininterrupta entre 23 de janeiro e 3 de julho de 2014 (162 dias). O intervalo de amostragem para cada série de tempo foi de 1 minuto. Para a análise das séries de tempo, foi utilizado o programa estatístico $R$ ( $R$ Core Team, 2013) e os pacotes "TSA" (Chan \& Ripley, 2012) e "WaveletComp" (Roesch \& Schmidbauer, 2014).

\section{Resultados e Discussão}

$\mathrm{Na}$ Fig. 2 são mostradas as séries de tempo de contagens, precipitação (acumulada em 24 h), e as componentes u (leste para oeste) e v (sul para norte) da velocidade do vento. Nessa figura, os dados são brutos, i.e., são apresentados como coletados, apenas os dados de precipitação foram acumulados em períodos de $24 \mathrm{~h}$ para facilitar a visualização. Como esperado, a comparação visual da variação das contagens e da precipitação mostra claramente que existe uma forte correlação entre a ocorrência de chuvas e o aumento das contagens, mostrando que o radon washout pode ser inferido a partir da observação da variação da radiação de fundo local. Com o detector utilizado, nesse local a contagem da radiação de fundo é de cerca de 35000 contagens/min, durante o radon washout esse valor pode chegar a mais de 45000 contagens/minuto, um aumento de cerca de $30 \%$. Mesmo chuvas de baixa intensidade (< $5 \mathrm{~mm} / \mathrm{dia}$ ) são capazes de produzir o radon washout. Com relação à influência do vento, a comparação das séries de tempo de contagens e das componentes da velocidade do vento não indica de maneira evidente a existência de correlação entre variações na intensidade do vento e contagens.

Os espectros de potência, calculados através da FFT das séries de tempo de contagens, precipitação e intensidade da velocidade do vento são mostrados na Fig.3. Nessa figura vemos que existe uma frequência dominante de 1 ciclo/dia nas séries de tempo de contagens e intensidade da velocidade do vento. No caso das contagens, essa periodicidade é devida ao ciclo de aquecimento e resfriamento do solo, que por sua vez influencia o processo de exalação do radônio pelo solo (Hosoda et al., 2007). Para a intensidade do vento, o ciclo diurno é explicado pelo ciclo diário de aquecimento e resfriamento da atmosfera. Para a precipitação, em função de sua característica mais aleatória de ocorrência, não foi registrado a existência de uma frequência dominante que descreve a ocorrência de chuvas para o local onde esse estudo foi realizado. Para as contagens e intensidade de velocidade do vento observamos periodicidades de fraca amplitude com frequência inferior a 0,2 ciclos por dia que provavelmente correspondem às passagens de sistemas frontais sobre a região, que favorecem a formação de precipitação e consequentemente do radon washout e intensificação dos ventos.

A correlação cruzada entre as séries de tempo de contagens, precipitação, e intensidade da velocidade do vento é mostrada na Fig. 4. Os resultados são exibidos com relação ao valor do lag expresso em dias. 


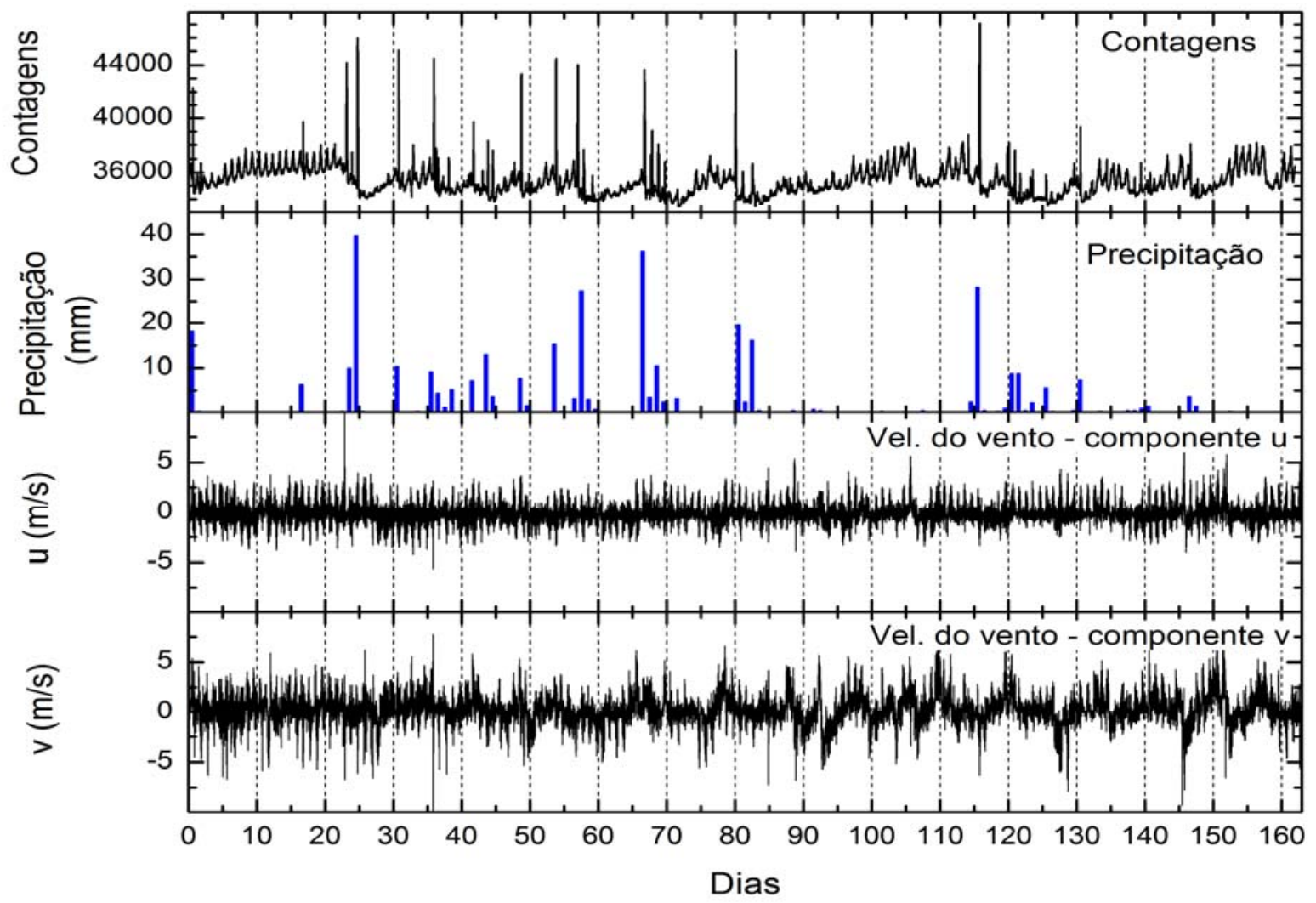

Figura 2 - Séries de tempos de contagens, precipitação e intensidade do vento Início, 23/01/2014; término 04/07/2014 (162 dias). Amostragem de 1 minuto. Dados de precipitação (em azul) são acumulados para períodos de 24 hara melhor visualização.

Na Fig. 4, os resultados indicam existir correlação entre a ocorrência de precipitação e o número de contagens corroborando os resultados exibidos na Fig. 2. É interessante observar que o maior valor da correlação entre contagens "lags" a precipitação em 1 dia, ou seja, o número de contagens devido ao radon washout atinge o seu valor máximo 1 dia após a ocorrência de precipitação, diminuindo nos dias seguintes em concordância com a meia vida do decaimento radioativo do ${ }^{222} \mathrm{Rn}$. Notamos também que não existe uma correlação do tipo causa-efeito entre as séries de tempo de contagens e intensidade da velocidade do tempo. A fraca correlação observada para lags maiores que cinco dias provavelmente é o resultado indireto da passagem de sistemas frontais que ocorrem em média a intervalos de 7 a 10 dias. Nessa situação, a passagem desses sistemas pode levar a formação de chuvas e a ocorrência do radon washout e a intensificação de ventos, sem que exista uma relação direta entre eles. Um efeito esperado do vento seria a dissipação do radônio, resultando em uma correlação negativa, o que não foi observado.

Os espectros de ondaletas das séries de tempo de tempo são mostradas na Fig. 5. A inspeção dos resultados nessa figura mostra a existência de variações intermitentes com período de 1 dia e de alguns dias na série de tempo de contagens. A série de tempo de precipitação apresenta periodicidades relacionadas à passagem de sistemas frontais (períodos $>5$ dias). Para a intensidade da velocidade do vento, observamos a componente de variação diurna discutida anteriormente. A comparação do espectro de ondaletas das séries de tempo de contagens e intensidade da velocidade do vento parece não indicar a existência de correlação de entre essas duas séries.

\section{Conclusão}

A monitoração da radiação de fundo, especialmente em áreas onde existem atividades humanas relacionadas à utilização de radioisótopos e a produção de energia em usinas nucleares é obviamente de grande importância. Porém, essa monitoração pode ser comprometida em situações quando, por exemplo, a concentração local de ${ }^{222} \mathrm{Rn}$ varia de maneira inesperada. Essas variações têm o potencial de mascarar aumentos nos níveis de radiação local causados por vazamentos, e outros tipos de acidentes. Nesse estudo, procuramos determinar se o vento pode ter um efeito inverso e causar uma diminuição dos níveis de radiação causados pelo ${ }^{222} \mathrm{Rn}$, especialmente relacionados a fenômeno do radon washout. Os resultados obtidos indicam que o vento não afeta de maneira importante os níveis de radiação de fundo local, indicando que durante eventos de radon washout, não ocorre dissipação significante desse gás por ventos locais. 


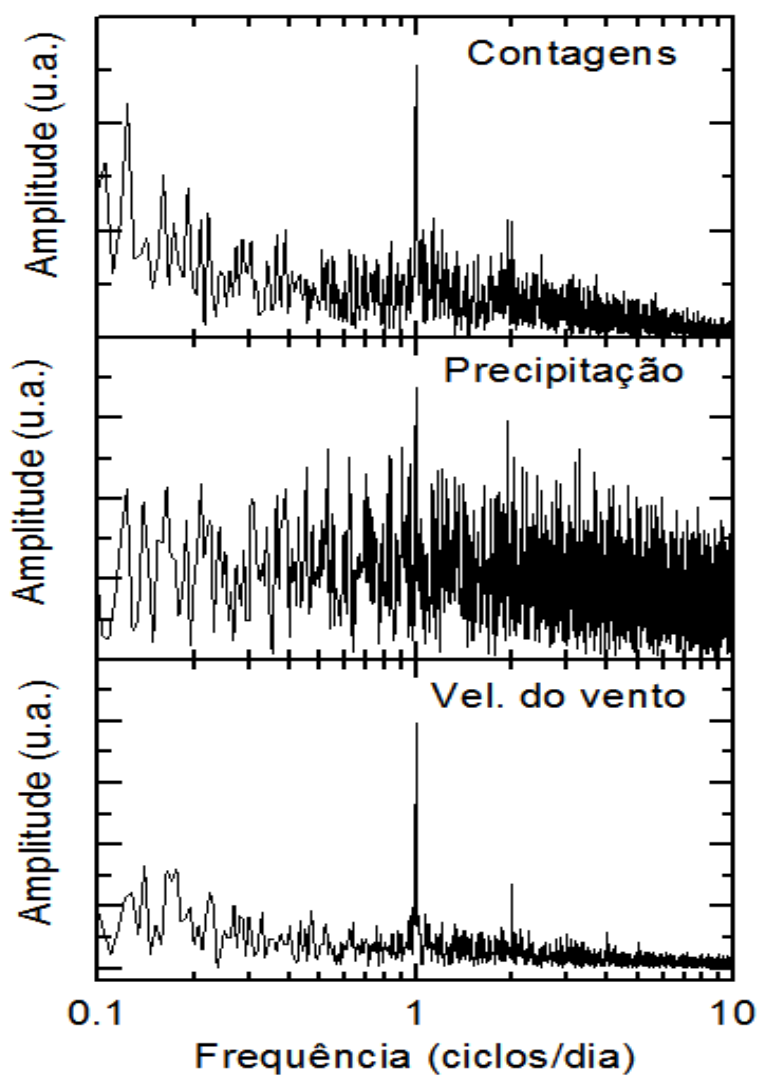

Figura 3 - Espectro de potência das séries de tempo de contagens, precipitação e intensidade do vento.

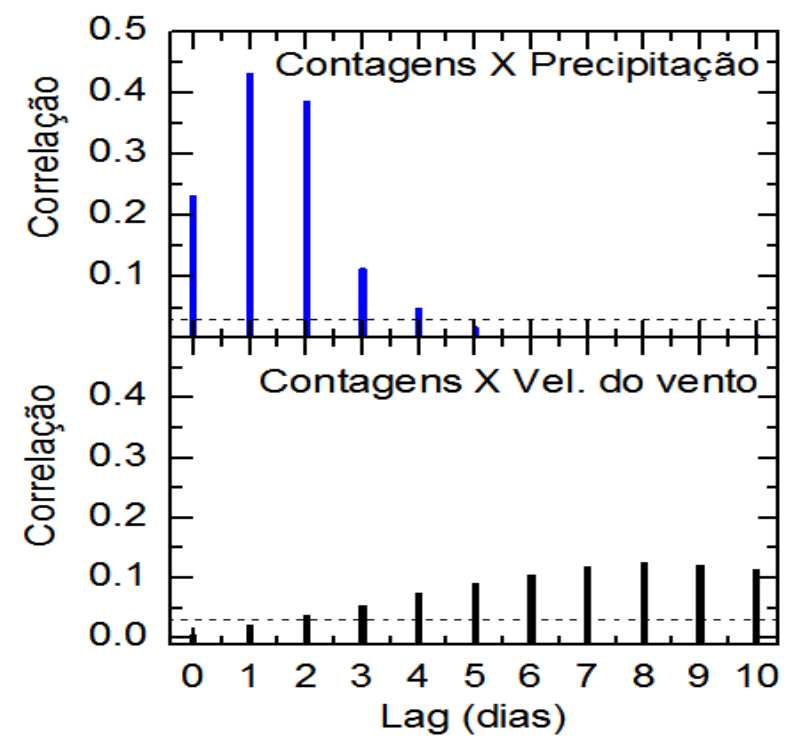

Figura 4 - Correlação cruzada entre as séries de tempo de contagens e precipitação e contagens e intensidade da velocidade do vento. A linha tracejada indica o nível de confiança de $95 \%$.

\section{Agradecimentos}

Os autores agradecem à CAPES e ao CNPq pelo auxílio financeiro e ao DCTA, ITA e IAE-ACA pelo apoio na realização deste trabalho

\section{Referências}

BHANDARI N, \& RAMA, 1963, Study of atmospheric washout processes by means of radon decay products, J. Geophys. Res., 68, 3823-3826.

CHAN KS \& RIPLEY B. 2012. TSA. Time Series Analyis. $\mathrm{R}$ package version 1.01. http://CRAN.Rproject.org/package $=$ TSA

CPRM, 2010, Serviço Geológico do Brasil, Geodiversidade do Estado de São Paulo: programa de geologia do Brasil levantamento da geodiversidade, São Paulo: CPRM.

DENMAN AR, GROVES-KIRKBY CJ , CROCKETT R \&. PHILLIPS, PS. 2004. Periodicity in domestic radon time series; Evidence for earth Tides. 11th International Congress of the International Radiation Protection Association 23-28 May, 2004 Madrid, Spain.

GROVES-KIRKBY CJ, DENMAN AR, CROCKETT RGM, PHILLIPS PS \& GILLMORE GK. 2006. Identification of tidal and climatic influences within domestic radon timeseries from Northamptonshire, UK. Sci. Tot. Environ. 367:191-202.

HOSODA M, SHIMO M, SUGINO M, FURUKAWA M \& FUKUSHI M. 2007. Effect of Soil Moisture Content on Radon and Thoron Exhalation, Journal of Nuclear Science and Technology, 44:4, 664-672

MAGILL J, PFENNIG G, DREHER $R$ \& SÓTI $Z$. 2015.Karlsruher Nuklidkarte / Chart of the Nuclides. 9th edition. Nucleonica $\mathrm{GmbH}$, Eggenstein-Leopoldshafen 2015.

R CORE TEAM, 2013,. R: A language and environment for statistical computing. R Foundation for Statistical Computing, Vienna, Austria.URL http://www.Rproject.org/.

ROESCH A \& SCHMIDBAUER H. 2014. WaveletComp: Computational Wavelet Analysis. R package version 1.0. http://CRAN.R-project.org/package=WaveletComp

WILKENING M. 1990. Radon in the Environment, Elsevier, The Netherlands, 137pp.

WILLIAMS AG, ZAHOROWSKI W, CHAMBERS $S$, GRIFFITHS A, HACKER JM, ELEMENT A \&AND WERCZYNSKI S. 2011. The vertical distribution of radon in clear and cloudy daytime terrestrial boundary layers. J. Atmos. Sci. 68 (1), 155-174 

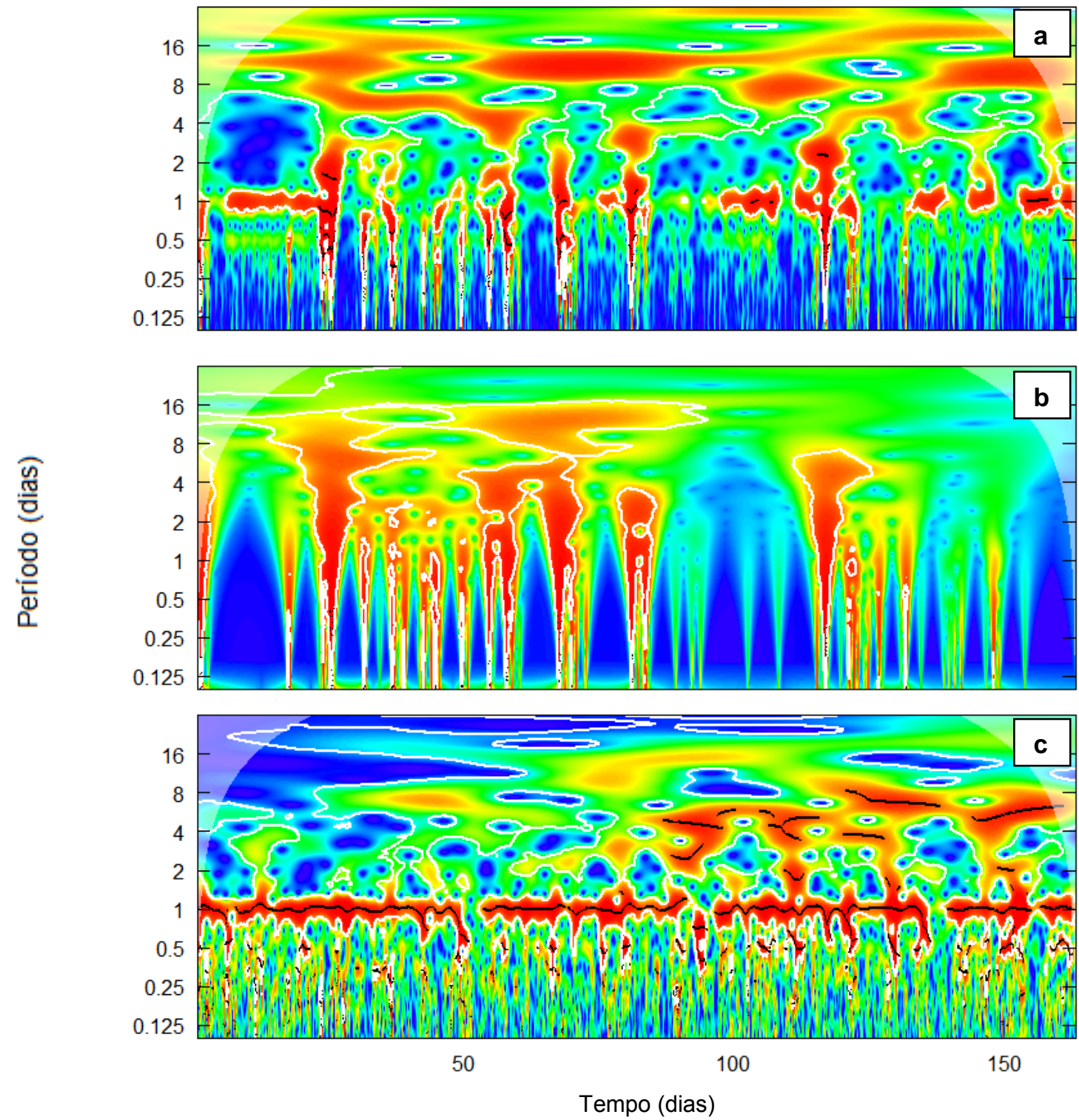

Figura 5 - Espectro de ondaletas das series de tempo de contagens (a), precipitação (b) e intensidade de velocidade do vento (c). Cores quentes indicam maior potência; cores frias, menor potência. 